Gazi University
Journal of Science
http://dergipark.gov.tr/gujs

\title{
Low-Error Reconfigurable Fixed-Width Multiplier for Image Processing Applications
}

\author{
Jean Jenifer Nesam JEYAKUMAR ${ }^{(1)}$, Sivanantham SATHASIVAM* ${ }^{(10}$ \\ School of Electronics Engineering, Vellore Institute of Technology, Vellore-632 014, India.
}

Highlights

- Design of low-error fixed-width multiplier for image processing applications.

- New area-efficient algorithm for unsigned multiplication.

- Performed in three different modes.

- Reconfigurable from fixed-width to exact multiplier.

- Image multiplication evaluates the performance of the multiplier.

Article Info

Received: $21 / 06 / 2018$

Accepted: 28/07/2019

\section{Keywords}

Fixed-Width multiplier Reconfigurable multiplier NBPR algorithm

Image processing applications

\begin{abstract}
Compensating the error using additional circuitry is mandatory in a low-error fixed-width multiplier. Instead of compensating the error, reconfiguring n-bit fixed-width multiplier to $n / 2$ bit error-free full-width multiplier using decomposed multiplication is proposed in this paper. The decomposed block multiplication using an area-efficient New Bit Pair Recoding (NBPR) algorithm in fixed-width mode shows a relatively lesser truncation error than existing truncated multipliers. Reconfigurable $16 \times 16$ NBPR multiplier in three different modes $(8 \times 8,16 \times 8,16 \times 16)$ with a fixed 16 -bit product is verified on the TSMC $65 \mathrm{~nm}$ CMOS standard cell library. The experimental results show that the NBPR multiplier consumes a lesser area than standard Booth multipliers. Evaluating the proposed multiplier in imaging shows improved PSNR with minimal error compared to other fixed-width multipliers.
\end{abstract}

\section{INTRODUCTION}

Fixed width multiplication is a mandatory arithmetic computation in digital signal and image processing applications. The fixed-width multipliers get two n-bit inputs and produce the n-bit product. This can be achieved by truncating a few least significant parts of partial products (PPs) matrix [1,2]. The PostTruncated (PT) fixed-width multiplier round off the product after the completion of multiplication. PT provides better accuracy but occupies a large area. The Direct-Truncated (DT) fixed-width multiplier directly truncates the n-least significant PPs without error compensation. DT occupies less area but provides less accuracy. For an area-efficient, low error fixed-width multiplier, the truncation error is compensated using various techniques. Kidambi et al. [3] introduced a simple constant bias technique that reduced the error by adding constant bias to the retained adder cells. But the error can still be reduced. Later, the Variable Correction Technique (VCT) [4] and data-dependent compensation to compensate for the error have been developed [5-8]. In VCT, the unused PP bits are used to define the compensation vector. The calculated compensation vector is added with the product to reduce the error. Juang et al. [9] developed efficient carry generating circuits that generate the carry and feed the carry as an input to the non-truncated adder cells to compensate for the error. Flexible error based multiplier and multiply-add design have been developed for area reduced error-resilient applications [10]. Self-tuned error compensation is an efficient method to reduce the truncation error [11-13]. The area reduction multiplication using an approximation for image processing application is developed in [14,15]. 
All error compensated multipliers provided in this literature require an additional complex circuit to find the amount of compensation vector. Albeit, it shows relatively more error for image processing applications. In this paper, the fixed-width multiplier performs without error compensation and provides the product accuracy better than error compensated fixed-width multipliers. The multiplier is reconfigurable to the exact multiplier using a control bit that provides distortion less application. In an inexact mode, the proposed multiplier eliminates the lower bits of the least decomposed block and the non-truncated higher bits are added to the higher blocks. This helps to reduce the truncation error with a slight increment in the area.

This area overhead is balanced by using an efficient New Bit Pair Recoding (NBPR) algorithm. NBPR reduces the PP row height without computing Negative Encoding (NE) and Sign Extension (SE). NE and $\mathrm{SE}$ are the common terms in existing bit pair recoding algorithms such as Booth [16-18] that incurred additional PP row which in turn occupies more area, power, and delay. NBPR generates the PP based on the number of 1's in 4-bit recoded multiplier groups. The performance benefits of NBPR is given in section 2. Finally, the developed multiplier is applied to image processing applications such as two different image multiplication and image color conversion. The behavioral simulation models are developed in Matlab to evaluate the performance of multiplier in imaging. The quality of the image is analyzed with its Peak Signal to Noise Ratio (PSNR) and compared with various truncated multipliers.

The rest of the paper is organized as follows; section 2 describes the performance benefits of the NBPR multiplier over existing algorithm based multiplication. Section 3 gives the detail about reconfigurable multiplication. The introduced error due to truncation is analyzed in section 4. Section 5 for results and comparison and section 6 explains the suitability of the proposed multiplier for different image processing applications. Section 7 concludes the paper.

\section{NEW BIT PAIR RECODING (NBPR) ALGORITHM}

\subsection{The NBPR Recoding}

Let us consider, ' $\mathrm{Y}$ ' is the multiplier and NBPR recodes the multiplier $\mathrm{Y}$ as follows:

$Y_{j}=\left\{y_{2 i+3}, y_{2 i+2}, y_{2 i+1}, y_{2 i}\right\}$

where, $i=2 j-2$ and $j=1,2,3 \ldots \frac{n}{4}$.

In Equation (1), the ' $\mathrm{j}$ ' represents the number of recoded 4-bit groups. Based on the requirement of ' $\mathrm{j}$ ', ' $\mathrm{i}$ ' takes the value defined by $2 \mathrm{j}-2$. For an example, 12-bit multiplier defines $\mathrm{j}=1,2,3$ and $\mathrm{i}=0,2,4$. For each $\mathrm{j}$ and $i$ value the multiplier is recoded as follows in Equation (2)

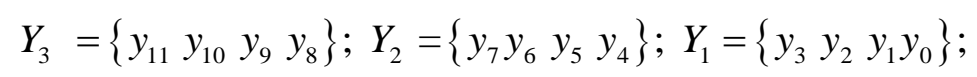

Based on the recoded group the NBPR selects the PDVs for PP reduction.

\subsection{PP Reduction Using NBPR Algorithm}

NBPR recoded 4-bit group performs the PP reduction using the diverse modules namely, PDV (PDV) generation, select logic (recoding), Merged PP addition. The following sub-section details each phase individually: 


\subsubsection{PDV Generation}

This module takes in two binary numbers for multiplication and recodes the multiplier operand as a nonoverlapped 4-bit group. Prior to multiplication, this module pre-defines a few values. Considering ' $\mathrm{X}$ ' as the multiplicand $\left(X=x_{n-1} x_{n-2} \ldots x_{2} x_{1} x_{0}\right.$ ) and the PDVs are assumed as follows:

1. The representation of ' 0 ' in Table 1 is computed $0 \times X$.

2. The other representations namely A, B, C, D are obtained by concatenating 0 's in a different position with multiplicand as below Equation (3),

$$
\begin{aligned}
& A=000 x_{n-1} x_{n-2} \ldots x_{2} x_{1} x_{0} \\
& B=00 x_{n-1} x_{n-2} \ldots x_{2} x_{1} x_{0} 0 \\
& C=0 x_{n-1} x_{n-2} \ldots x_{2} x_{1} x_{0} 00 \\
& D=x_{n-1} x_{n-2} \ldots x_{2} x_{1} x_{0} 000
\end{aligned}
$$

3. Adding ' $A$ ' and ' $B$ ' to generate ' $E$ ' value

4. The summed up value is left-shifted twice $((A+B)<<2)$ and represented as ' $F$ '

The values of A, B, C, D resemble the bit combinations of " 0001 ", " 0010 ", " 0100 " and " 1000 " respectively and the values are generated by replacing the 1's position with the multiplicand and without changing the 0 's position. The " 0011 " bit combination is generated as the result of adding ' $A$ ' and ' $B$ '. The bit combination "1100" is left-shifted twice to attain "0011". The computed PDVs are listed in Table 1 and the generated PPs using the PDVs are provided in Table 2.

\begin{tabular}{|c|c|c|c|c|c|}
\hline \multicolumn{2}{|c|}{ Recoded bits } & \multirow[b]{2}{*}{ Z1 } & \multicolumn{2}{|c|}{ Recoded bits } & \multirow[b]{2}{*}{$\mathrm{Z} 2$} \\
\hline$y_{i+1}$ & $y_{i}$ & & $y_{i+3}$ & $y_{i+2}$ & \\
\hline 0 & 0 & 0 & 0 & 0 & 0 \\
\hline 0 & 1 & A & 0 & 1 & $\mathrm{C}$ \\
\hline 1 & 0 & $\mathrm{~B}$ & 1 & 0 & $\mathrm{D}$ \\
\hline 1 & 1 & $E$ & 1 & 1 & $\mathrm{~F}$ \\
\hline
\end{tabular}

Table 1. PDVs of NBPR algorithm

\begin{tabular}{|l|l|l|}
\hline Bit combination & Operation & Representation \\
\hline 0000 & $0 \times X$ & 0 \\
\hline 0001 & \multirow{3}{*}{$\begin{array}{l}\text { Replace the 1's position by } \\
\text { the multiplicand }\end{array}$} & $\mathrm{A}$ \\
\cline { 3 - 3 } 0010 & & $\mathrm{~B}$ \\
\cline { 3 - 3 } 0100 & C \\
\hline 1000 & Add "0001" and "0010" & $\mathrm{D}$ \\
\hline 0011 & 2-bit left shift of "0011" & $\mathrm{F}$ \\
\hline 1100 & & \\
\hline
\end{tabular}

Table 2. PDV selection using the NBPR Algorithm

\subsubsection{Merged Addition}

The two sets of 4-bit recoded group generates two $(n+3)$-bit size intermediate PPs. Thereby, merged addition on intermediate PPs reduces the number of PP rows from $n$ to $n / 4$. The pictorial representation of pp reduction using the NBPR algorithm is shown in Figure 1. The flowchart representation of the NBPR multiplier is provided in Figure 2. However, the $8 \times 8$ NBPR multiplier generates two PPs, it skips the reduction phase and directly fed to the final addition phase. NBPR uses adder tree in Carry Save Addition (CSA) format for $n>8$-bit. The final addition stage uses Carry Propagate Adder (CPA) to add the sum and carry. 


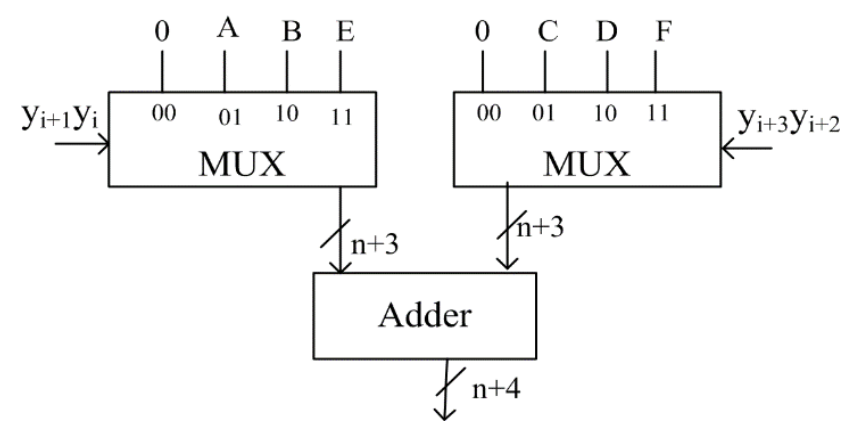

Figure 1. Merged PP generation and addition in the NBPR algorithm

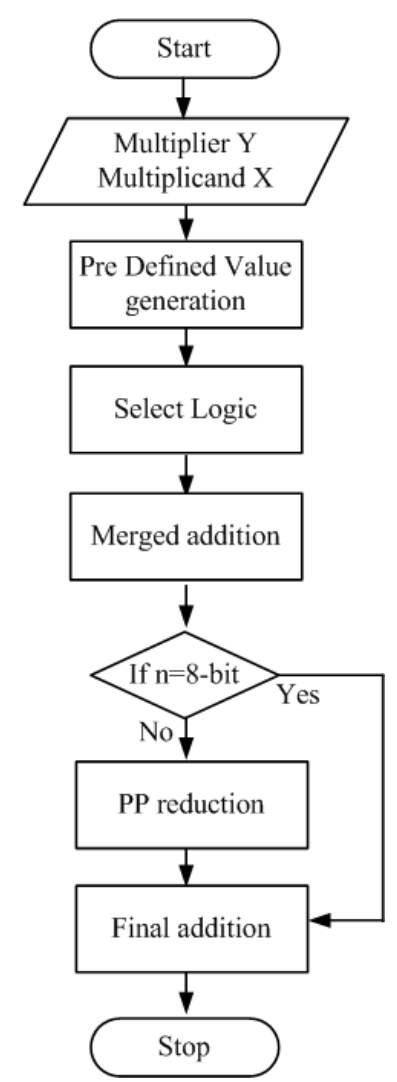

Figure 2. Flowchart for NBPR multiplier

\subsection{Realization of NBPR Algorithm for $n=8$-Bit Multiplication}

Consider two unsigned binary numbers $\mathrm{X}=10101100$ and $\mathrm{Y}=11101001$. Multiplication $\mathrm{X}$ is realized using the NBPR algorithm in the following steps. The diagrammatic representation of the NBPR algorithm for 8bit multiplication is given in Figure 3.

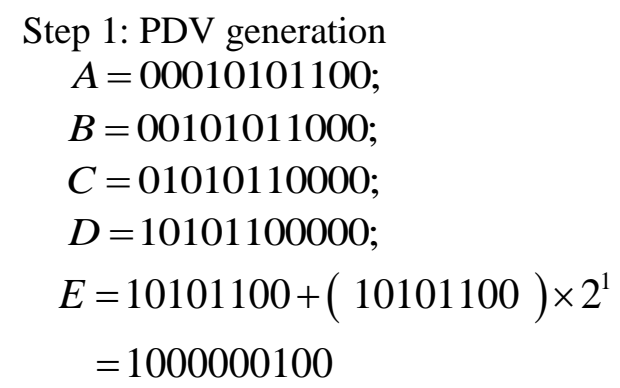




$$
F=E<<2=100000010000
$$

Step 2: Select Logic (Recoding)

$$
\begin{aligned}
& Y_{1}=y_{3} y_{2} y_{1} y_{0}=1001 \\
& Y_{2}=y_{7} y_{6} y_{5} y_{4}=1110
\end{aligned}
$$

MUX based selection of predefined value for the recoded bits

$$
\begin{aligned}
\text { MUX1 } & = \begin{cases}Z 1=A=00010101100 & y_{1} y_{0}=01 \\
Z 2=D=10101100000 & y_{3} y_{2}=10\end{cases} \\
M U X 2 & =\left\{\begin{array}{cc}
Z 1=B=00101011000 & y_{5} y_{4}=10 \\
Z 2=F=100000010000 & y_{7} y_{6}=11
\end{array}\right.
\end{aligned}
$$

Step 3: Merged Addition/PP reduction $(\mathrm{Z} 1+\mathrm{Z2})$

$$
\begin{aligned}
& P P 1=11000001100 ; \\
& P P 2=100101101000 ;
\end{aligned}
$$

Step 4: final Addition

The second PP (PP2) is placed 4-bit left to the first PP (PP1) and Carry Propagate Adder (CPA) is applied for the final addition to producing a 16-bit product

Final product $=P P 1+4-$ bit left shifted $P P 2$

$$
\begin{aligned}
& =11000001100+(100101101000) \times 2^{4} \\
& =1001110010001100
\end{aligned}
$$

\subsection{Decomposed 16x16 Multiplier Using 8x8 NBPR Multiplier}

The decomposed $16 \times 16$ multiplication split the 16-bit operand into two 8-bit sub-blocks and requires four $8 \times 8$ NBPR multiplication as provided in Figure 4 to compute the $16 \times 16$ multiplier. After the sub-block multiplication, the appropriate blocks are added and the addition of overlapping bits is performed as shown in Figure 5.

\subsection{Final Addition}

The final addition is computed via Carry Propagating Addition (CPA). The CPA length of Wallace depends on the number of stages involved in the PP reduction phase. If $\mathrm{S}$ is the number of stages and $N$ is the number of bits in the operands, the final CPA length of Wallace is defined as in Equation (4)

for $3 \leq \mathrm{N} \leq 5$,

$$
\text { CPA length } \text { Wallace }=(2 \times N)-2-S
$$

and if $\mathrm{N}>5$

$$
\text { CPA length } \text { Wallace }=(2 \times N)-1-S
$$




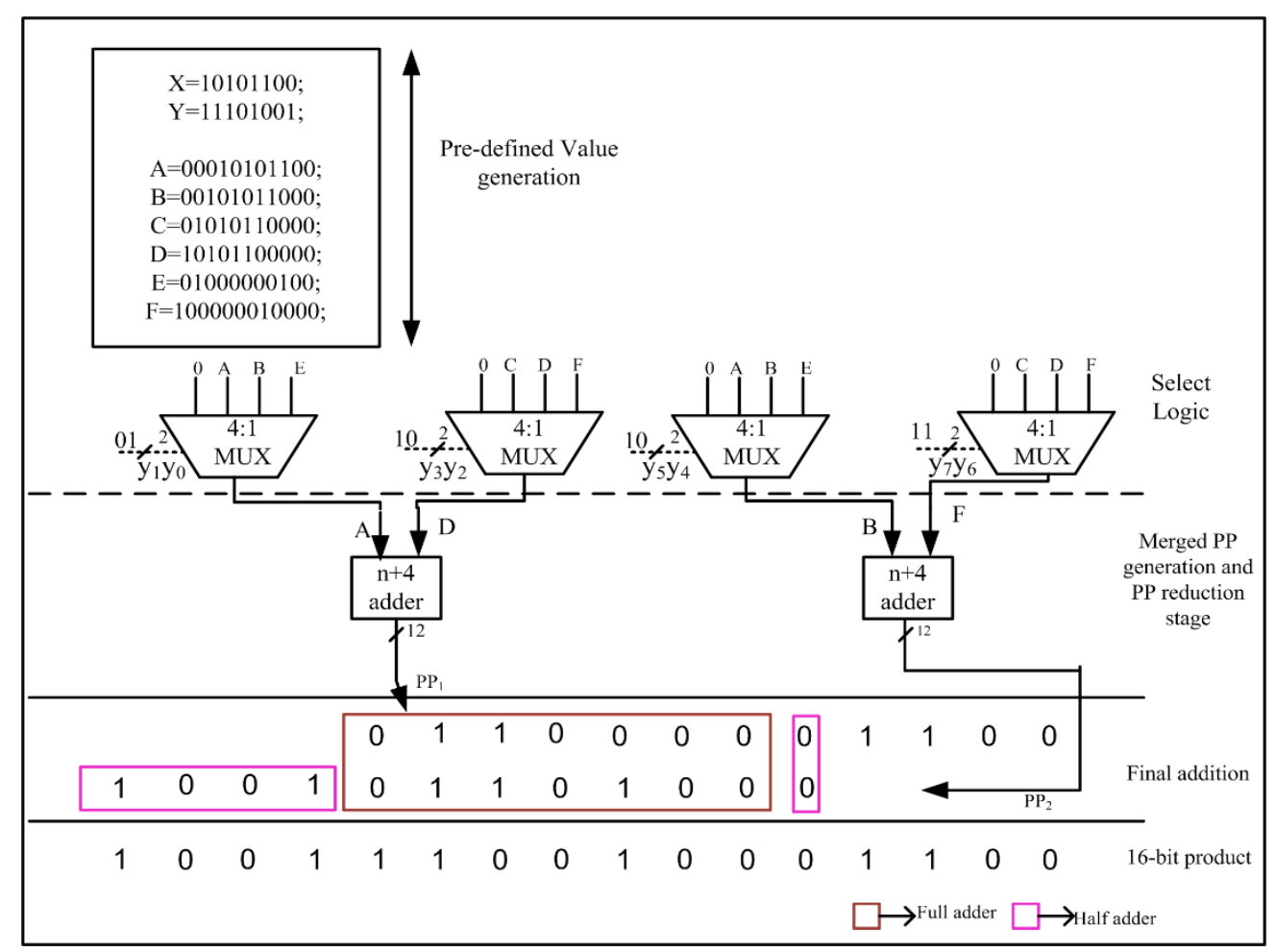

Figure 3. $8 \times 8$ multiplication using NBPR algorithm

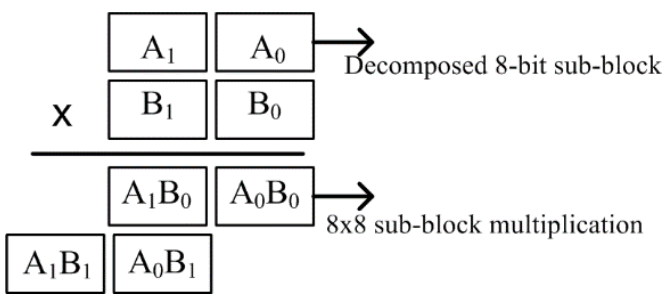

Figure 4. Decomposed 16x16 NBPR multiplier

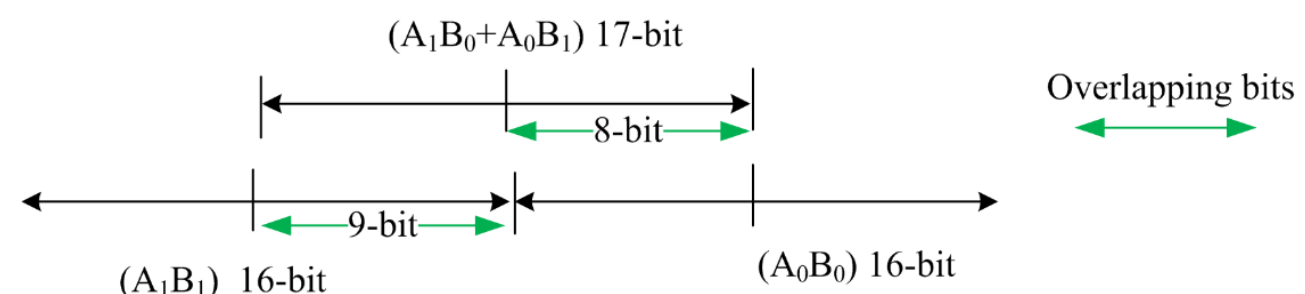

Figure 5. Final addition for $16 \times 16$ NBPR multiplier

A direct implementation of N-bit multiplier or Dadda N-bit multiplier's final CPA length is defined as in Equation (5)

CPA length $_{\text {Dadda }}=2 N-2$

The proposed NBPR multiplier has 20\% less CPA length and CPA size is determined by Equation (6)

CPA length $_{\text {Proposed }}=17 i+7$ 
where,

$$
i=\left\{\begin{array}{cl}
\sim 0.23 & \text { for } 8 \times 8 \text { multiplier } \\
1 & \text { for } 16 \times 16 \text { multiplier } \\
2 & \text { for } 24 \times 24 \text { mutiplier } \\
3 & \text { for } 32 \times 32 \text { multiplier }
\end{array}\right.
$$

\section{RECONFIGURABLE FIXED WIDTH MULTIPLIER}

Small-sized and high accuracy applications need $8 \times 8$ exact multiplication, whilst, the large bit-sized errorresilient application uses the $16 \times 8$ or $16 \times 16$ inexact multiplication based on the requirement. A fixed width multiplier is the utmost common multiplier used in image processing applications. Many truncated methods available to maintain the $16 \times 16$ multiplier with the 16 -bit product. Directly truncate the least part of the partial product matrix is the simple method that achieves more area reduction with large accuracy loss. This accuracy loss is compensated in various ways using variable or constant correction compensation vectors. These compensation methods consume more area.

This reconfigurable multiplier that can perform both exact and inexact multiplication without any area overhead. Distraction less image applications achieve in $8 \times 8$ exact mode albeit, the other two modes performing the application with less accuracy loss compared to existing truncated multiplier. The new reconfigurable multiplier is developed in this paper that can perform both exact multiplications without error and truncated multiplication with less error. In this proposed multiplier performs $n \times n, n \times \frac{n}{2}$ and $\frac{n}{2} \times \frac{n}{2}$ multiplication with the fixed n-bit product. This reconfigurability is achieved by using decomposed block multiplication and a 1-bit control bit. The decomposed multiplier plane for $16 \times 16$ multiplier using four $8 \times 8$ NBPR multiplier is shown in Figure 6 .

In NBPR based fixed-width multiplier computes the multiplication in three different modes and the length of the data is modified based on the application requirement. Selecting differently sized sub-block multiplication as given in Figure 7, the reconFigured multiplication is performed. If A and B are two 16bit operands, the control bits and the corresponding mode of multiplication are given in Table 3.

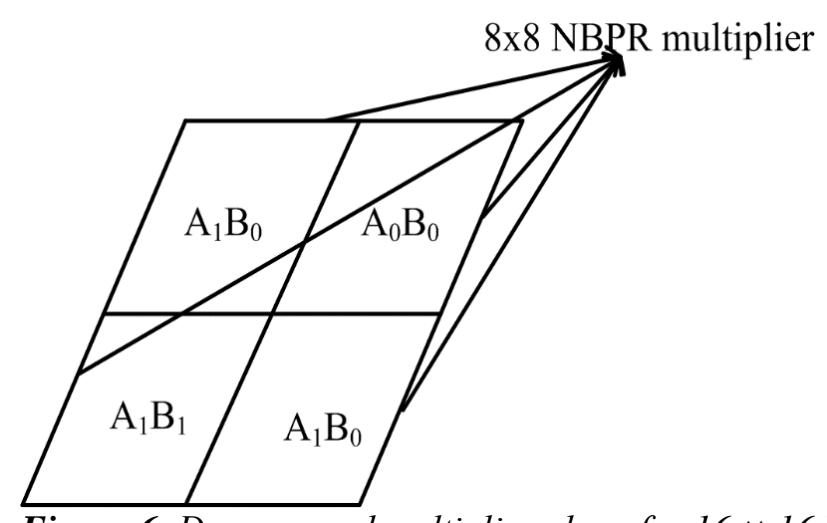

Figure 6. Decomposed multiplier plane for $16 \times 16$ NBPR multiplier

Table 3. Control Bits and Corresponding Mode Selection

\begin{tabular}{|c|c|c|c|c|}
\hline \multirow[b]{2}{*}{ Mode } & \multirow[b]{2}{*}{ Control bit } & \multicolumn{2}{|c|}{ Input size } & \multirow[b]{2}{*}{ Output size } \\
\hline & & A & B & \\
\hline 1 & 1 & 16-bit & 16-bit & \multirow{3}{*}{ 16-bit } \\
\hline 2 & 0 & 8-bit & 8-bit & \\
\hline 3 & 0 & 16-bit & 8-bit & \\
\hline
\end{tabular}


In the proposed fixed-width multiplier, the output product size is fixed to 16-bit. The new NBPR multiplier can be performed as a $16 \times 16$ fixed-width multiplier ie). $16 \times 16$ multiplication with a 16-bit product in mode 1 . In mode 2 , it performs an exact $8 \times 8$ multiplication i.e). $8 \times 8$ multiplier with the 16-bit product, and truncated $16 \times 8$ multiplier with 16 -bit product in mode 3 .

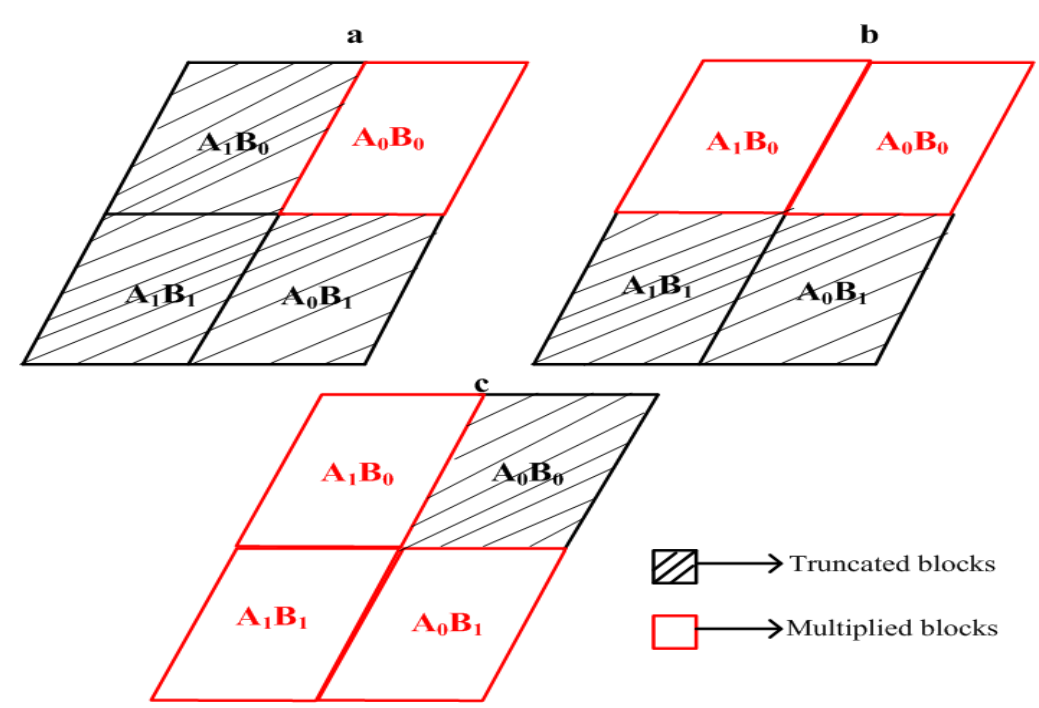

Figure 7. Three modes of computation a). $8 \times 8$ full-width multiplier b). $16 \times 8$ truncated multiplier c). $16 \times 16$ truncated multiplier

As shown in Figure 8, the 1-bit control bit selects the upper or lower half of the operand B values. If the control bit is ' 0 ', the 2:1 multiplexer selects the lower half of the $B$ operand $B_{0}$ and $\left(A_{0} B_{0}\right)$ NBPR $8 \times 8$ multiplication is performed. $\left(A_{0} B_{0}\right)$ multiplication is considered as a $8 \times 8$ exact multiplier with 1the 6-bit product. Addition of $\left(A_{0} B_{0}\right)$ and uthe pper half of the $\left(A_{1} B_{0}\right)$ provides 16-bit truncated results for $16 \times 8$ multiplication. If the control bit is ' 1 ', the upper half of the $\mathrm{B}$ operand is $B_{1}$ selected and $\left(A_{1} B_{1}\right),\left(A_{0} B_{1}\right)$ block multiplications are computed in mode 2. Direct $\left(A_{1} B_{0}\right)$ multiplication, $\left(A_{1} B_{1}\right)$ and $\left(A_{0} B_{1}\right)$ blocks are added accordingly to provide fixed width $16 \times 16$ multiplication in mode 3 .

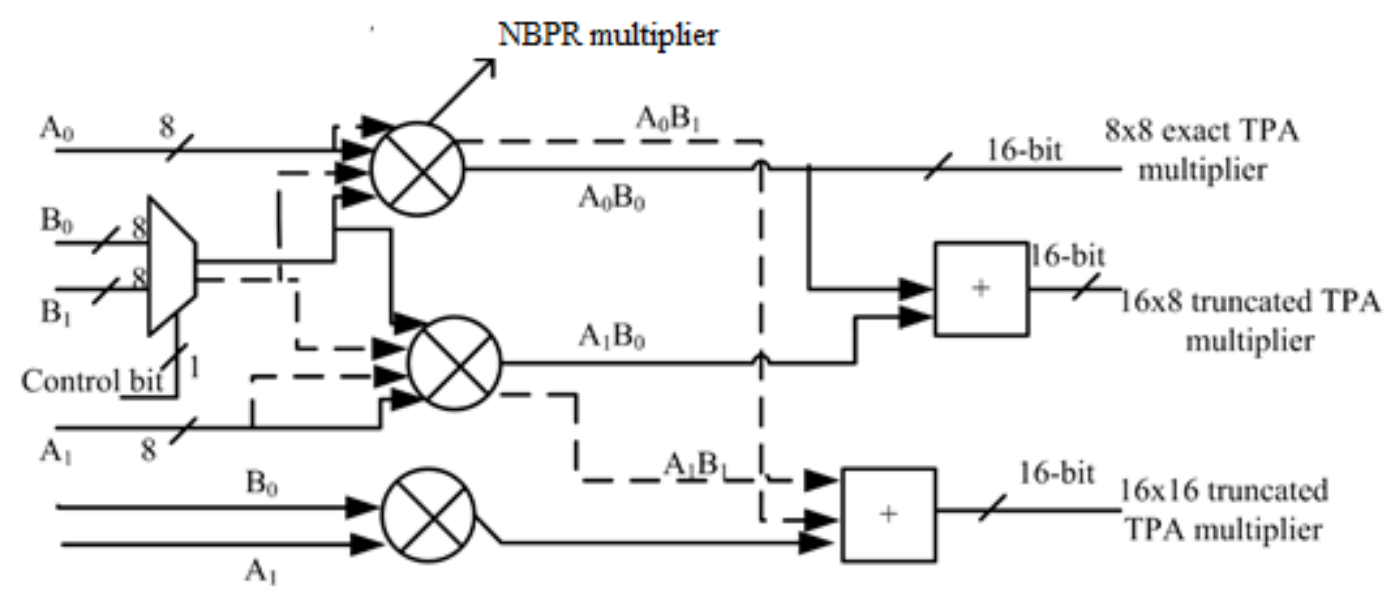

Figure 8. Mode selection of NBPR multiplier

\section{ERROR ANALYSIS}

The $16 \times 16$ multiplication is achieved by using four $8 \times 8$ sub-blocks. All sub-block multiplications are performed in parallel. The half of the product of each multiplied sub-blocks are overlapped to the next 
higher block and the truncation is performed only in the final addition stage. Keeping the overlapping bits and truncate the lower non-dependent bits help to reduce the truncation error. The generic product form of various modes of decomposed NBPR multiplier is given as in Equation (7).

$$
\begin{aligned}
& P_{8 \times 8}=P_{A_{0} B_{0}} ; \\
& P_{16 \times 8}=P_{A_{1} B_{0}}+P_{A_{0} B_{0}} ; \\
& P_{16 \times 16}=P_{A_{1} B_{1}}+P_{\left(A_{1} B_{0}+A_{0} B_{1}\right)}+P_{A_{0} B_{0}}
\end{aligned}
$$

Each $P_{A_{x} B_{y}}$ and $x, y \in(0,1)$ in different modes refers one $8 \times 8 \mathrm{NBPR}$ multiplier. The error due to truncation in each mode is analysed in the following sub-section.

\subsection{NBPR $8 \times 8$ Exact Multiplication}

In $8 \times 8$ multiplication mode, it operates as an exact multiplier as given in Equation (8).

$$
P_{8 \times 8}=P_{A_{0} B_{0}}=\sum_{i=0}^{7} a_{i} \times \sum_{i=0}^{7} b_{j}
$$

where $\left(A_{0} B_{0}\right)$ is the $8 \times 8$ sub-block and $a_{i}, b_{j} \in(0,1)$ are individual bits inside the sub-block. The $8 \times 8$ multiplication provides 16 - bit product without error. The maximum error is computed as the difference between the exact product and the truncated product. Since $8 \times 8$ is an exact multiplier, the truncation part is considered as equivalent to exact product and the maximum error is equal to zero and the value is computed as shown in Equation (9)

$$
\varepsilon_{\max _{T P A \& \times 8}}=\max _{A, B}\left(\left|P_{\text {exact }}-P_{\text {truncated }}\right|\right)=0 \text {. }
$$

\subsection{NBPR $16 \times 8$ Truncated Multiplier}

In this mode, the multiplier gets two different sizes operand $(16,8)$ for multiplication. The exact multiplication of $16 \times 8$ provides the 24-bit product. After the computation, truncating partial part of $P_{\left(A_{0} B_{0}\right)}$ , the product size is fixed to 16-bit. The error limit in this mode is calculated as follows in Equation (10)

$$
\begin{aligned}
\mathrm{P}_{(16 \times 8)}=\mathrm{P}_{\mathrm{A}_{1} \mathrm{~B}_{0}}+\mathrm{P}_{\mathrm{A}_{0} \mathrm{~B}_{0}} & =\sum_{\mathrm{i}=8}^{15} \mathrm{a}_{\mathrm{i}} \times \sum_{j=0}^{7} b_{j}+\sum_{\mathrm{i}=0}^{7} \mathrm{a}_{\mathrm{i}} \times \sum_{j=0}^{7} b_{j} \\
& =\sigma_{\text {high }}+\sigma_{\text {low }} \\
\mathrm{P}_{16 \times 8(\mathrm{r})} & =\sigma_{\text {high }}+\sigma_{\text {low(upper half }) .}
\end{aligned}
$$

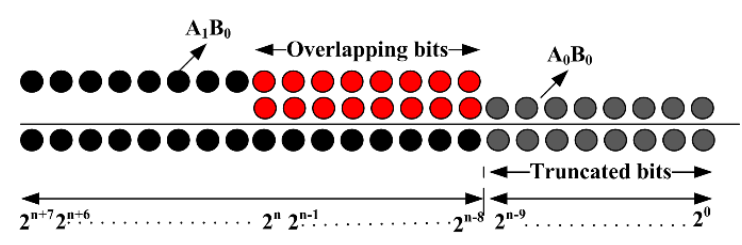

Figure 9. The $\left(n \times \frac{n}{2}\right)$ multiplication with $n-$ bit product $(n=16-$ bit $)$ 
The error limits can be computed from Figure 9, and the truncation error $\left(\varepsilon_{t r}\right)$ of $16 \times 8$ the truncated multiplier is bounded as given in Equation (11),

$2^{0} \leq \varepsilon_{t r} \leq 2^{n-9}$

where $n=16-$ bit.

\subsection{NBPR 16x16 Fixed-Width Multiplier}

In this mode, the $16 \times 16$ multiplier discards the lower $\left(A_{0} B_{0}\right)$ block without computation and truncate a few parts of the product $\left(A_{0} B_{1}+A_{1} B_{0}\right)$ to fix the output product size to 16-bit.

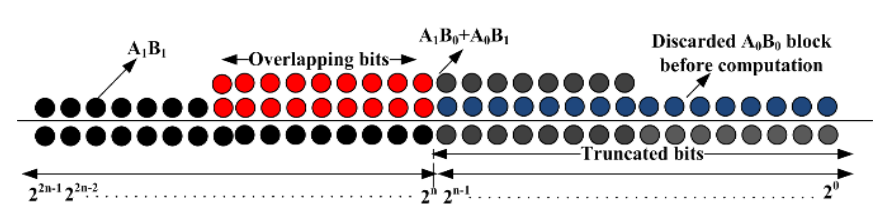

Figure 10. The $(n \times n)$ multiplication with $n$ - bit product $(n=16-$ bit $)$

The truncation performed as given in Figure 10 and the truncation error limit in this mode is computed as follows in Equation (12)

$$
\begin{aligned}
P_{16 \times 16_{\text {freed widh }}} & =P_{A_{1} B_{1}}+P_{\left(A_{1} B_{0}+A_{0} B_{1}\right)} \\
& =\sum_{\mathrm{i}=8}^{15} \mathrm{a}_{\mathrm{i}} \times \sum_{j=8}^{15} b_{j}+\left(\sum_{\mathrm{i}=8}^{15} \mathrm{a}_{\mathrm{i}} \times \sum_{j=0}^{7} b_{j}+\sum_{\mathrm{i}=0}^{7} \mathrm{a}_{\mathrm{i}} \times \sum_{j=8}^{15} b_{j}\right) \\
& =\sigma_{\text {high }}+\sigma_{\text {mid(upper half })} .
\end{aligned}
$$

\section{PERFORMANCE EVALUATION}

The functionality of the presented multiplier is verified on Modelsim 6.5b. After the functional verification, the multiplier is synthesized using Cadence's RC RTL compiler in 180 and $65 \mathrm{~nm}$ technology. The separate TCL and SDC script are written for each mode. The performance of the proposed design is individually evaluated in the following sections.

\subsection{Analysis of NBPR Multiplier}

Detailed area, power and delay analysis of NBPR multiplier with other bit pair recoding algorithm is provided in this section. The Modified Booth Encoding (MBE) is the common algorithm for area-efficient multiplication. MBE reduces the PP rows with the computation of NE and SE terms. Since NE and SE incurred more resources, many designs have been developed with the elimination of NE and SE. The negation of NE requires additional circuitry and its computations are highly complex. Even though the SE prevention techniques have been adopted, two SE bits till required in multiplication. From, the theoretical analysis of various Booth multipliers with NBPR claims the superiority of the proposed design.

The righteousness of theoretical analysis has been proven by its experimental results. Each module in the NBPR algorithm namely PDV generation ('E' value), recoder logic, PP reduction and final addition for 8, 16-bit multiplication is performed in 180 and $65 \mathrm{~nm}$ technology and the results are provided in Table 4 . The excel of the NBPR algorithm in terms of area, power, and speed is verified by computing the design for 
generating one bit of PP. The compared experimental results for generating one PP bit using various algorithms are provided in Table 5 and is the evidence that the NBPR uses only $72.5 \%$ less area and $18 \%$ less power with $34 \%$ increased speed when compared to the standard booth multipliers.

Table 4. Synthesis report of various phases of NBPR multiplier (180 and 65nm)

\begin{tabular}{|c|c|c|c|c|c|c|c|c|c|c|}
\hline \multirow{2}{*}{\multicolumn{2}{|c|}{$\begin{array}{l}\text { Mode of } \\
\text { computation }\end{array}$}} & & \multicolumn{4}{|c|}{$\mathrm{n}=8$-bit } & \multicolumn{4}{|c|}{$\mathrm{n}=16$-bit } \\
\hline & & $\mathrm{nm}$ & \#cells & $\begin{array}{l}\text { Area } \\
\left(\mu m^{2}\right)\end{array}$ & $\begin{array}{l}\text { Power } \\
(\mu W)\end{array}$ & $\begin{array}{l}\text { Delay } \\
\text { (ns) }\end{array}$ & \#cells & $\begin{array}{l}\text { Area } \\
\left(\mu m^{2}\right)\end{array}$ & $\begin{array}{l}\text { Power } \\
(\mu W)\end{array}$ & $\begin{array}{l}\text { Delay } \\
\text { (ns) }\end{array}$ \\
\hline \multirow{2}{*}{\multicolumn{2}{|c|}{$\begin{array}{l}\text { PDV } \\
\text { generation }\end{array}$}} & 180 & 25 & 719 & 82.832 & 0.056 & 59 & 981 & 101.189 & 0.637 \\
\hline & & 65 & 24 & 61 & 8.187 & 0.044 & 31 & 132 & 1.316 & 0.081 \\
\hline \multirow{4}{*}{ Recoder } & \multirow{2}{*}{$\begin{array}{l}\text { Select } \\
\text { logic }\end{array}$} & 180 & 113 & 2365 & 87.853 & 0.190 & 259 & 5409 & 870.269 & 2.173 \\
\hline & & 65 & 88 & 362 & 23.247 & 0.017 & 146 & 639 & 156.321 & 0.236 \\
\hline & \multirow{2}{*}{$\begin{array}{l}\text { merged } \\
\mathrm{pp}\end{array}$} & 180 & 23 & 283 & 9.345 & 0.052 & 30 & 336 & 49.267 & 0.527 \\
\hline & & 65 & 22 & 44 & 2.128 & 0.056 & 31 & 49 & 9.844 & 0.056 \\
\hline \multirow{2}{*}{\multicolumn{2}{|c|}{ PP reduction }} & 180 & \multicolumn{4}{|l|}{-} & 253 & 4697 & 624.573 & 0.564 \\
\hline & & 65 & \multicolumn{4}{|l|}{-} & 94 & 558 & 169.290 & 0.067 \\
\hline \multirow{2}{*}{\multicolumn{2}{|c|}{ Final adder }} & 180 & 18 & 233 & 8.559 & 0.052 & 156 & 2262 & 217.123 & 8.772 \\
\hline & & 65 & 18 & 36 & 1.891 & 0.051 & 25 & 221 & 72.295 & 0.786 \\
\hline
\end{tabular}

Table 5. Area/power/delay report on generating one PP bit using MBE and NBPR in $65 \mathrm{~nm}$

\begin{tabular}{|l|l|l|l|l|}
\hline Design & $\#$ cells & $\begin{array}{l}\text { Area } \\
\left(\mu m^{2}\right)\end{array}$ & $\begin{array}{l}\text { Power* } \\
(\mu \mathrm{W})\end{array}$ & $\begin{array}{l}\text { Delay } \\
(\mathrm{ps})\end{array}$ \\
\hline Standard Booth & 4 & $14(100 \%)$ & $0.967(100 \%)$ & $84(100 \%)$ \\
\hline Limberti [16] & 6 & $16(114.3 \%)$ & $0.795(84.7 \%)$ & $74(88 \%)$ \\
\hline Antelo [17] & 6 & $14(100 \%)$ & $0.735(83.4 \%)$ & $61(72.7 \%)$ \\
\hline NBPR & 3 & $10(72.5 \%)$ & $0.713(82 \%)$ & $56(66.7 \%)$ \\
\hline \multicolumn{5}{|c}{ *Power $=$ Leakage power+Dynamic power }
\end{tabular}

\subsection{Reconfigurable 16x16 NBPR Multiplier}

The area, power and delay consumption of reconfigurable NBPR multiplier is verified in this section. The Verilog code has been written for reconfigurable design as in Figure 8 and its functionality has been verified in Modelsim 6.5b simulator. After the functional verification, the design has been implemented on Cadence TSMC 180 and $65 \mathrm{~nm}$ with a standard cell library. The obtained results are provided in Table 6.

The area consumption of various truncated multipliers is compared with the NBPR multiplier in three different modes. The compared values on TSMC $180 \mathrm{~nm}$ are provided in Table 7 . The presented NBPR multiplier in $16 \times 16$ fixed-width multiplication mode occupies $33.15 \%$ less area when compared to standard Booth multiplier. In full-width mode $8 \times 8$ shows approximately $1 \%$ less area and in truncated $16 \times 8$ mode shows $54.65 \%$ less area compared to the standard multiplier. However, the NBPR multiplier occupies $15.5 \%$ more area compared to other direct truncated multipliers mentioned in the literature, due to the error reduced decomposed multiplication.

Table 6. Implementation details of NBPR multiplier on 180 and $65 \mathrm{~nm}$ TSMC technology

\begin{tabular}{|l|l|l|l|l|l|}
\hline Technology & Area $\left(\mu m^{2}\right)$ & Power $(\mu W)$ & Delay (ns) & PDP $(\mathrm{fJ})$ & APP $\left(\mu m^{2} \cdot \mu W\right) 10^{5}$ \\
\hline $180 \mathrm{~nm}$ & 20654 & 1286.093 & 0.5 & 643.047 & 265.63 \\
\hline $65 \mathrm{~nm}$ & 3614 & 220.176 & 0.5 & 110.088 & 7.96 \\
\hline
\end{tabular}


Table 7. Area consumption of various truncated multipliers on 180nm technology

\begin{tabular}{|l|l|l|}
\hline \multirow{2}{*}{ Design } & Area $\left(\mu \mathrm{m}^{2}\right)$ & $\mathrm{n}=16$ \\
\cline { 2 - 3 } & $\mathrm{n}=8$ & 30896.219 \\
\hline Standard multiplier & 7092.164 & 15179.200 \\
\hline D-truncated & 3337.495 & 15179.200 \\
\hline K-G-As' [6] & 3299.660 & 16477.370 \\
\hline J-K-Cs' [1] & 3640.836 & 20688.631 \\
\hline K-Ss'[4] & 5604.888 & 16477.370 \\
\hline L.D. Vans' [3] & 3640.83 & $14011(16 \times 8) \mathrm{tr}$ \\
\hline \multirow{2}{*}{ Proposed } & \multirow{2}{*}{$7037(8 \times 8)$ exact } & $20654(16 \times 16) \mathrm{tr}$ \\
\hline
\end{tabular}

Table 8. Max Error ( $\left.\varepsilon_{\max }\right)$ for various $16 \times 16$ fixed width multiplier

\begin{tabular}{|l|l|l|l|}
\hline \multirow{2}{*}{ Design } & \multirow{2}{*}{ Algorithm } & \multicolumn{2}{|c|}{$\varepsilon_{\max }$} \\
\cline { 3 - 4 } & & Bit width (n) \\
\cline { 3 - 4 } & & $\mathrm{n}=8$ & $\mathrm{n}=16$ \\
\hline D-truncated & - & 1793 & 983040 \\
\hline Post-truncated & - & 128 & 32768 \\
\hline K-G-As' [6] & 2's complement & 1280 & 786432 \\
\hline J-K-Cs' [1] & Booth & 514.94 & 219316.22 \\
\hline K-Ss' [4] & Sign-magnitude & 263.03 & 111083.52 \\
\hline L.D Vans' [3] & Baugh-Wooley & 441 & 220245 \\
\hline Yuan-Ho Chen [2] & 2's complement & 384.9 & 169181 \\
\hline Proposed & \multirow{2}{*}{ NBPR } & 0 & $128(16 \times 8)$ \\
\cline { 3 - 4 } & & $05537(16 \times 16)$ \\
\hline
\end{tabular}

\subsection{Maximum Error $\left(\varepsilon_{\max }\right)$ Computation}

The maximum error $\left(\varepsilon_{\max }\right)$ is theoretically computed as the difference between original results and actual results by applying its maximum value. The $\varepsilon_{\max }$ for $16 \times 16$ multiplier in different modes with various truncation depth $(\tau)$ value is given in Table 8 . Compared to directly truncated (D-truncated) multiplier, the NBPR multiplier shows $93.33 \%$ reduction in error while, K-G-As', J-K-Cs', K-Ss', L.D Vans' and YuanHo Chens' designs have error improvement of 20\%, 77.69\%, 88.7\%, 79.63\%, and $82.79 \%$ respectively. Hence the NBPR multiplier shows significant error improvement, compared to direct truncate and other truncating multipliers.

\section{APPLICATIONS}

\subsection{Image Multiplication}

The behavioral model of the multiplier is developed in Matlab and two $512 \times 512$ images are multiplied with different $\tau$ values. The $\tau$ value and the corresponding multiplied images are given in Figure 11. The quality of the resultant images is verified by using Peak Signal to Noise Ratio (PSNR). The PSNR is calculated by the Equation (13).

PeakSNR $=$ PSNR $\left(\right.$ image $_{\text {tr }}$, image $\left._{\text {exact }}\right)$. 
Table 9. PSNR of a multiplied image using NBPR multiplier

\begin{tabular}{|l|l|l|l|}
\hline \multirow{2}{*}{ Images } & \multicolumn{3}{|l|}{ PSNR $(\mathrm{dB})$} \\
\cline { 2 - 4 } & $\tau=0$ & $\tau=8$ & $\tau=16$ \\
\hline image1 & $\infty$ & 54.5716 & 17.3624 \\
\hline image2 & $\infty$ & 54.42 & 15.3881 \\
\hline image3 & $\infty$ & 54.6148 & 14.2321 \\
\hline
\end{tabular}
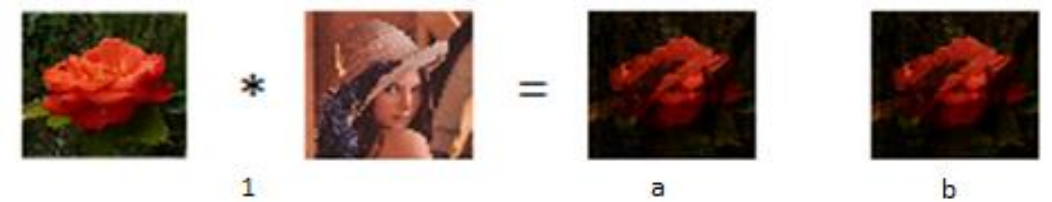

b
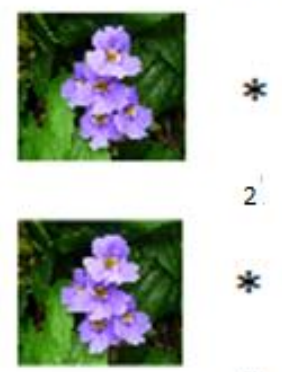

3

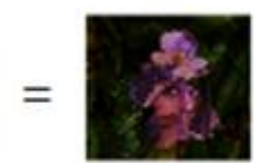

a

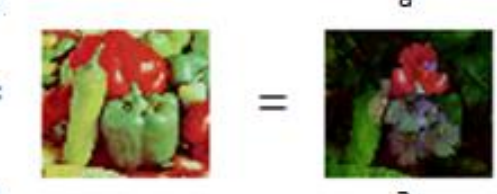

a b

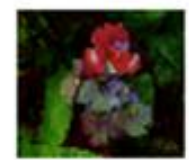

b

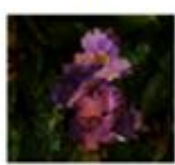

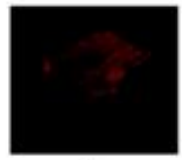

c

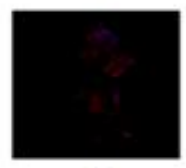

c

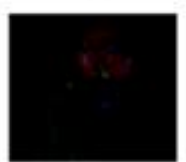

c

Figure 11. Multiplied images 1). image1 2). image2 3). image3 with a). $\tau=0$ b). $\tau=8 c$ ). $\tau=16$

The PSNR is computed between the NBPR multiplier applied image and the exact multiplier applied image. The PSNR shows that the presented NBPR multiplier performs with better image quality. The PSNR values are given in Table 9. The various truncated multiplier based image multiplication in Table 10 is evident for the excellence of the proposed multiplier in imaging.

Table 10. PSNR comparison

\begin{tabular}{|l|l|l|}
\hline \multirow{2}{*}{ Design } & \multicolumn{2}{l|}{ PSNR (dB) } \\
\cline { 2 - 3 } & $\tau=8$ & $\tau=16$ \\
\hline DT & 28.81 & 9.64 \\
\hline PT & 33.45 & 11.34 \\
\hline K-G-As' [6] & 38.20 & 12.48 \\
\hline J-K-Cs' [1] & 39.46 & 13.65 \\
\hline K-Ss' [4] & 39.76 & 11.08 \\
\hline L.D Vans' [3] & 34.01 & 10.30 \\
\hline Yuan-Ho Chens' [2] & 31.84 & 10.98 \\
\hline Proposed & 54.61 & 14.23 \\
\hline
\end{tabular}

\subsection{Image Color Space Conversion (RGB to Gray)}

The Red, Green and Blue values in the RGB color image has the integer range from 0 to 255 . Multiplying $\mathrm{R}, \mathrm{G}$, and $\mathrm{B}$ value with different coefficient and adding the multiplied values together provides different color space. The NBPR multiplier in $8 \times 8$ exact multiplication mode is well suited for image color space conversion such as RGB color space to grayscale transformation. The RGB image is converted to a grayscale image using below Equation (14). The color-converted images from RGB to gray is given in Figure 12. 
Gray Image $=0.2989 \times \mathrm{R}+0.587 \times \mathrm{G}+0.114 \times \mathrm{B}$
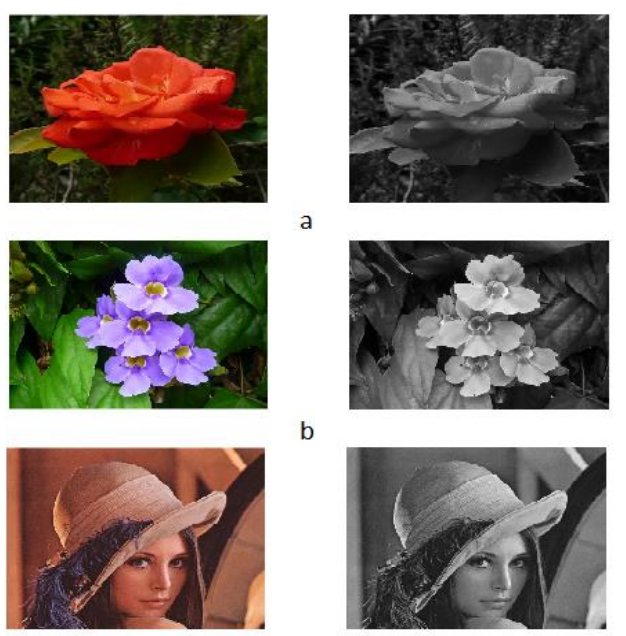

b

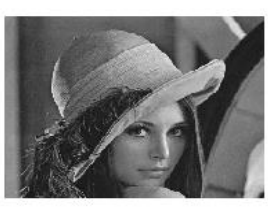

Figure 12. RGB to Gray a. flowerl b. flower 2 c. Lena

\section{CONCLUSION}

Reconfigurable exact and inexact decomposed multipliers using a new algorithm for image processing applications have been presented in this paper. Based on the selection of different input sizes, the multiplier reconFigured from the exact $8 \times 8$ multiplier to inexact $16 \times 8$ and $16 \times 16$ multiplier. The error-free computation in $8 \times 8$ mode has provided the distortionless image quality, howbeit, the inexact other two modes $(16 \times 8,16 \times 16)$ have provided $93.33 \%$ lesser error compared to directly truncated multipliers. Moreover, the exact $8 \times 8$ NBPR multiplier is consumed $33.15 \%$ lesser area compared to standard MBE multipliers. Finally, the detailed performance analysis of various color image applications showcased the outperformance of a new multiplier with minimal degradation of image quality when compared to existing fixed-width multipliers. This makes the proposed reconfigurable NBPR multiplier more amicable for image processing applications.

\section{CONFLICTS OF INTEREST}

No conflict of interest was declared by the authors.

\section{REFERENCES}

[1] Jou, S.J. and Wang, H.H., "Fixed-width multiplier for DSP application", Proc. IEEE International Symposium on Computer-Aided Control System Design , 318-332, (2000).

[2] Chen, Y.H., Lu, C.W., Chiang, H.C., Chang, T.Y., Hsia, C., "A low-error statistical fixed-width multiplier and its applications," International Symposium on Instrumentation \& Measurement, Sensor Network and Automation (IMSNA), Sanya, 39-43, (2012).

[3] Van, L.D., Yang, C.C., "Generalized low-error area-efficient fixed width multipliers," IEEE Transactions on Circuits and Systems-I: Regular Papers, 52(8): 1608-1619, (2005).

[4] King, E.J., Swartzlander, E.E. Jr., "Data-dependent truncation scheme for parallel multipliers," Proc. 31st Asilomar Conference Signals, Systems, and Computers, 2: 1178-1182, (1997).

[5] Chen, Y-H., Li, C.Y., Chang T.Y., "Area-Effective and Power-Efficient Fixed-Width Booth Multipliers Using Generalized Probabilistic Estimation Bias", IEEE Journal on Emerging and Selected Topics in Circuits and Systems, 1: 277-288, (2011). 
[6] Kidambi, S.S., El-Guibaly, F. Antoniou, A., "Area-efficient multipliers for digital signal processing applications," IEEE Transactions on Circuits and Systems II Analog and Digital Signal Processing, 43(2): 90-94, (1996).

[7] Petra, N., Caro, D.D. Garofalo, V, "Truncated binary multipliers with variable correction and minimum mean square error", Transactions on Circuits and Systems I: Regular Papers, 57(6): 13121325, (2010).

[8] Wey, I.C., Wang, C.C, "Low-error and hardware-efficient fixed width multiplier by using the dualgroup minor input correction vector to lower input correction vector compensation error", IEEE Transactions on Very Large Scale Integration (VLSI) Systems, 20(10): 1923-1928, (2012).

[9] Juang, T.B., Hsiao, S.F., "Low-error carry-free fixed-width multipliers with low-cost compensation circuits", IEEE Transactions Circuits Systems II, 52(6): 299-303, (2005).

[10] Caro, D.D., Petra, N., Strollo, A.G.M., "Fixed-width multipliers and multipliers-accumulators with min-max approximation error",Transactions on Circuits and Systems I: Regular Papers , 60(9): 2375$2388,(2013)$.

[11] Wey, I.C., Peng, C.C., Liao, F.Y., "Reliable low-power multiplier design using fixed width replica redundancy block", IEEE Transactions Very Large Scale Integr. (VLSI) Systems, 23(1): 78-87, (2015).

[12] Drane, T.A., Rose, T.M., Constantinides, G.A., "On the Systematic Creation of Faithfully Rounded Truncated Multipliers and Arrays," IEEE Transactions on Computers, 63(10): 2513-2525, (2014).

[13] Joshi, P.U., Deshmukh, R.B., Gudur, V., "Self-compensation scheme for truncation error in fixed width multipliers," IET Circuits, Devices \& Systems, 12(1): 55-62, (2018).

[14] Venkatachalam, S., Ko, S.B., "Design of Power and Area Efficient Approximate Multipliers," IEEE Transactions on Very Large Scale Integration (VLSI) Systems, 25(5): 1782-1786, (2017).

[15] Sertbaş, A., El-Abdallah, H., Karabiber, F., "A Fast Multiplier Hardware Design for Interval Arithmetic", Istanbul University-Journal of Electrical and Electronics Engineering, 6: 169-174, (2012).

[16] Lamberti, F., "Reducing the computation time in (short bit-width) twos complement multipliers," ,IEEE Transactions on Computers, 60(2): 148-156, (2011).

[17] Antelo, E., Montuschi, P., Nannarelli, A., "Improved 64-bit Radix-16 Booth Multiplier Based on Partial Product Array Height Reduction," IEEE Transactions on Circuits and Systems I: Regular Papers, 64(2): 409-418, (2017).

[18] Ding, J., Li, S., "A Modular Multiplier Implemented With Truncated Multiplication," IEEE Transactions on Circuits and Systems II: Express Briefs, 65(11): 1713-1717, (2018). 